\title{
Análise crítica dos escores de avaliação de gravidade da fibrose cística: Estado da arte
}

\author{
Critical analysis of scoring systems used in the assessment of Cystic Fibrosis \\ severity: State of the art
}

CAMILA ISABEL DA SILVA SANTOS, JOSE DIRCEU RIBEIRO, ANTONIO FERNANDO RIBEIRO, GABRIEL HESSEL

\begin{abstract}
Este trabalho descreve, compara e analisa os escores de avaliação de gravidade da fibrose cística publicados na literatura nos últimos anos, e contextualiza o surgimento e o objetivo de cada um deles. Através de levantamento bibliográfico, foram encontrados 16 escores de gravidade da fibrose cística: 8 escores de avaliação clínica, 5 radiográficos, 2 tomográficos e 1 proposta de escore cintilográfico. Apesar das críticas e controvérsias quanto à objetividade e ao realismo das informações obtidas através desses escores, sua aplicação, ao longo da história da fibrose cística, mostrou-se útil por contribuir para um maior entendimento da doença e da efetividade das diversas terapêuticas.
\end{abstract}

J Bras Pneumol 2004; 30(3) 286-298

Descritores: Fibrose cística. Índice de gravidade de doença. Revisão de leitura.

\section{INTRODUÇÃO}

A fibrose cística (FC) ou mucoviscidose é uma desordem letal, hereditária, autossômica recessiva, mais comum em caucasianos, em que são identificadas cerca de mil mutações diferentes. 0 defeito compromete a proteína reguladora da condutância trans-membrana, o que resulta em um aumento na viscosidade das secreções glandulares e diminuição do clearance mucociliar(1). A manifestação clínica da doença difere entre os pacientes, com variação no grau de comprometimento pulmonar e gastro-intestinal. 0 quadro respiratório é apontado como a causa mais comum de morte ${ }^{(2)}$. A deterioração pulmonar progressiva decorre de inflamação, retenção de muco, infecção crônica por Staphylococcus aureus e Pseudomonas, hiper-reatividade brônquica, hiper insuflação, instabilidade e obstrução das vias aéreas ${ }^{(3)}$. Não há consenso sobre a
This study carries out a descriptive and comparative analysis of the various types of cystic fibrosis severity scores described in the literature and contextualizes the origin and objective of each. A total of 16 scoring systems were found: 8 are used predominantly for clinical evaluation, 5 for radiographic findings, 2 for tomographic findings and 1 for scintigraphic findings. Despite the criticism and controversy regarding these instruments of assessment, they have contributed to a better understanding of the disease and to the development of more effective therapeutic procedures.
Key words: Severity of illness index. Cystic fibrosis. Review literature. antibioticoterapia ideal para o tratamento ${ }^{(4)}$, mas o controle das infecções pulmonares é parte importante do conjunto de cuidados, assim como a melhora do clearance da secreção brônquica, a correção do defeito de má-absorção e o suporte nutricional $^{(1)}$. A individualidade de cada paciente também é considerada na terapêutica, sendo a aderência ao tratamento e a gravidade da doença descritos, desde os primeiros trabalhos, como elementos importantes no prognóstico ${ }^{(5,6,7,8)}$.

Essa variabilidade clínica, assim como o reconhecimento da FC como uma entidade importante e freqüente na clínica médica, determinou o desenvolvimento de sistemas de escores de avaliação de sua gravidade, os quais contribuem para a caracterização e avaliação do curso da doença, e retratam seu histórico, além das peculiaridades fenotípicas das diferentes populações. Os escores foram elaborados através 
da análise e comparação dos sinais e sintomas apresentados pelos pacientes ${ }^{(9)}$, e o surgimento de cada um deles teve relação direta com o entendimento da doença no contexto científico. Os escores de gravidade da fibrose cística (EGFC) são usados há décadas para avaliar a extensão da lesão pulmonar, comparar a gravidade clínica dos pacientes, avaliar os efeitos das intervenções terapêuticas e estimar o prognóstico. No entanto, não há consenso com relação ao escore ideal ${ }^{(10,11,12)}$. Tanto os escores clínicos como os radiológicos e os tomográficos serão discutidos neste estudo, pois são muito considerados e amplamente aplicados nos grandes centros de atendimento da doença (Quadro1).

Foi feita uma revisão da literatura (Medline) dos diferentes sistemas de escores para avaliação de gravidade da fibrose cística, bem como uma análise crítica, descritiva e comparativa dos diversos escores existentes.

\section{ESCORES DE AVALIAÇÃO}

0 levantamento bibliográfico permitiu encontrar 16 EGFC publicados e citados em conceituados estudos, sendo 8 escores clínicos, 5 radiológicos, 2 tomográficos e 1 cintilográfico, cada qual inserido em um momento histórico de compreensão

\begin{tabular}{|c|c|c|c|c|}
\hline & ESCORE & ANO & AVALIAÇÂO & FONTE \\
\hline 1 & Shwachman Score & 1958 & clínica & 5 \\
\hline 2 & Doershuk Score* & 1964 & clínica & 6 \\
\hline \multirow[t]{2}{*}{3} & Simplified Cystic & & & \\
\hline & Fibrosis Scale - SCS & 1971 & clínica & 7 \\
\hline 4 & Taussig Score - NIH & 1973 & clínica & 8 \\
\hline 5 & Chrispin Norman Score & 1974 & radiológica & 17 \\
\hline 6 & Brasfield Score & 1979 & radiológica & 10 \\
\hline 7 & Scintigraphic score & 1980 & cintilográfica & 18 \\
\hline 8 & Huang Score & 1981 & clínica & 19 \\
\hline 9 & C-N Score $*$ & 1982 & radiológica & 22 \\
\hline 10 & S-B Score * & 1987 & clínica & 23 \\
\hline 11 & Nathanson Score & 1991 & tomográfica & 24 \\
\hline 12 & Bhalla Score & 1991 & tomográfica & 25 \\
\hline 13 & Wisconsin Score & 1993 & radiológica & 26 \\
\hline 14 & Northern Score & 1994 & radiológica & 27 \\
\hline 15 & Matouk Score* & 1997 & clínica & 29 \\
\hline 16 & Kanga Score - CFCS & 1999 & clínica & 12 \\
\hline
\end{tabular}

Quadro 1. Escores de avaliação da gravidade na FC

\author{
Siglas e abreviaturas utilizadas neste trabalho: \\ FC: Fibrose cística \\ EGFC: escores de gravidade da FC \\ $\mathrm{FEV}_{1}$ : volume expiratório forçado no primeiro segundo \\ CVF: capacidade vital forçada
}

científica da doença, e todos com a intenção de retratar a gravidade e contribuir para a avaliação das intervenções terapêuticas.

Os primeiros relatos de casos e estudos de FC identificaram a morte da criança no período neonatal $^{(13,14)}$, idéia que acabou sendo modificada pelas demonstrações de trabalhos posteriores. Mclntosh ${ }^{(15)}$ fez o primeiro estudo prospectivo, acompanhando pacientes até os dez anos de idade para relacionar a evolução e a gravidade da doença. Em seguida, Royce(16) elaborou o primeiro conjunto de parâmetros clínicos para avaliação da doença, o qual incluiu apenas baqueteamento digital, tosse, freqüência respiratória e tolerância aos exercícios, e enfatizou apenas os sintomas, sem correlacioná-los com a gravidade ou extensão da lesão pulmonar ${ }^{(5)}$.

Foi no trabalho de Shwachman ${ }^{(5)}$, um estudo longitudinal com 105 fibrocísticos acompanhados durante cinco anos a partir do diagnóstico, que a questão da gravidade e da necessidade de um sistema de avaliação clínica fortaleceu-se, pois este evidenciou grande variação nos estágios da doença, independentemente da precocidade do diagnóstico. Identificou também a necessidade de inclusão da função pulmonar na avaliação. Esse sistema de avaliação clínica foi um marco no histórico científico da fibrose cística e até os dias atuais é respeitado e amplamente utilizado como instrumento clássico de avaliação da gravidade da doença ${ }^{(7,8,11,12)}$. 0 escore foi elaborado com 0 objetivo de comparar as manifestações clínicas entre os pacientes, detectar os efeitos do tratamento e contribuir para a determinação de critérios diagnósticos. Para tanto, esse escore tem quatro critérios maiores: atividade geral, nutrição, exame radiológico e avaliação física (Quadro 2). Cada critério varia de 5 a 25 pontos, e quanto menor a pontuação mais grave se encontra 0 paciente.

0 escore de Shwachman sofreu algumas críticas pela subjetividade e foi alterado por 
Doershuk, em 1964, que propôs um outro escore. Segundo este autor, este novo escore seria mais objetivo e poderia ser aplicado também em adolescentes e adultos. Manteve as quatro categorias de Shwachman, apenas incluindo alguns aspectos pertinentes aos adolescentes e adultos. Considerou uma variação de cinco pontos no resultado final da avaliação, e aconselhou sua aplicação em duplo cego e discussões dos casos polêmicos pelos examinadores. Em seu estudo, acompanhou as manifestações clínicas de pacientes em regime terapêutico por um período

\begin{tabular}{|c|c|c|}
\hline Pontuação & Atividade Geral & Achados Radiológicos \\
\hline 25 & $\begin{array}{l}\text { Atividade normal plena; joga bola; } \\
\text { vai a escola regularmente }\end{array}$ & campos pulmonares limpos \\
\hline 20 & $\begin{array}{l}\text { falta resistência e cansa ao final do dia; } \\
\text { boa freqüência escolar }\end{array}$ & $\begin{array}{l}\text { mínimas marcas de acentuação } \\
\text { broncovascular; enfisema primário }\end{array}$ \\
\hline 15 & $\begin{array}{l}\text { descansa voluntariamente durante o dia; } \\
\text { cansa facilmente após exercício; } \\
\text { freqüência escolar satisfatória }\end{array}$ & $\begin{array}{l}\text { enfisema leve; sinais de atelectasias; marcas } \\
\text { de aumento broncovascular }\end{array}$ \\
\hline 10 & $\begin{array}{l}\text { professor particular; repousa muito; } \\
\text { dispnéia após caminhada curta; }\end{array}$ & $\begin{array}{l}\text { enfisema moderado; áreas de atelectasias } \\
\text { difusas com áreas de infecção sobrepostas; } \\
\text { bronquiectasias mínimas }\end{array}$ \\
\hline 05 & ortopnéia; confinado a cama ou cadeira & $\begin{array}{l}\text { extensivas alterações com fenômenopulmonar } \\
\text { obstrutivo e infecção; aletectasias lobares e } \\
\text { bronquiectasias }\end{array}$ \\
\hline Pontuação & Nutriçâo & Exame Físico \\
\hline 25 & $\begin{array}{l}\text { mantém peso e altura próximo do } \\
\text { percentil } 25 \text {; bom tônus e massamuscular; } \\
\text { fezes bem formadas- quase normais }\end{array}$ & $\begin{array}{l}\text { normal: s/ tosse; FC e FR normais; pulmões } \\
\text { limpos; boa postura }\end{array}$ \\
\hline 20 & $\begin{array}{l}\text { peso e altura aproximadamente no } \\
\text { percentil de } 15 \text { a } 20 \text {; fezes levemente } \\
\text { anormais; tônus e massa musculartônus e } \\
\text { massa muscular satisfatórios }\end{array}$ & $\begin{array}{l}\text { Tosse rara ou "pigarro"; FC e FR normais no } \\
\text { repouso; mínimo enfisema; pulmões limpos; s/ } \\
\text { baqueteamento }\end{array}$ \\
\hline 15 & $\begin{array}{l}\text { peso e altura acima do percentil 3; fezes } \\
\text { usualmente anormais, volumosas e } \\
\text { pobremente formadas; tônus pobre e } \\
\text { massa muscular reduzida; pouca distensão } \\
\text { abdominal (se tiver) }\end{array}$ & $\begin{array}{l}\text { tosse ocasional (ao levantar pela manhã); FR } \\
\text { levemente elevada; enfisema suave; MV rude; } \\
\text { roncos localizados raramente; baqueteamento } \\
\text { precoce }\end{array}$ \\
\hline 10 & $\begin{array}{l}\text { peso e altura abaixo do percentil 3; fezes } \\
\text { pouco formadas, volumosas, ofensivamente } \\
\text { gordurosas; músculo fraco e massa } \\
\text { reduzida;distensão abdominal suave/ } \\
\text { moderada }\end{array}$ & $\begin{array}{l}\text { tosse freqüente, usualmente produtiva; } \\
\text { retração torácica; enfisema moderado; } \\
\text { deformidade torácica; roncos usualmente } \\
\text { presentes; baqueteamento } 2 / 3\end{array}$ \\
\hline 05 & $\begin{array}{l}\text { marcada má-nutrição; protuberância } \\
\text { abdominal abundante; fezes freqüentes, } \\
\text { volumosas, mau cheiro e gordurosas; } \\
\text { prolapso retal freqüente }\end{array}$ & $\begin{array}{l}\text { Tosse grave paroxística, taquipnéia e } \\
\text { taquicardia; alteração pulmonar extensa; } \\
\text { sinais de falência cardíaca direita; } 3 / 4 \\
\text { baqueteamento }\end{array}$ \\
\hline \multicolumn{2}{|r|}{ Classificação } & Pontos \\
\hline \multicolumn{2}{|r|}{ grave } & $<40$ \\
\hline \multicolumn{2}{|r|}{ moderado } & $55-41$ \\
\hline \multicolumn{2}{|r|}{ leve } & $70-56$ \\
\hline \multicolumn{2}{|r|}{$\begin{array}{l}\text { bom } \\
\text { excelente }\end{array}$} & $85-71$ \\
\hline & excelente & $100-86$ \\
\hline
\end{tabular}

Quadro 2. Escore de Shwachman 
de cinco anos. Como não registrou melhora no escore dos pacientes que apresentavam exame radiológico deteriorado, mesmo após a introdução da medicação, identificou uma nova questão: a importância do tratamento precoce das manifestações pulmonares ${ }^{(6)}$.

Em 1971, a partir das críticas surgidas aos escores, Cooperman criou uma forma simplificada, baseada no modelo do teste de Apgar, a Simplified Cystic Fibrosis Scoring Scale (SCS), com o objetivo de indicar os efeitos das medicações e tratamentos no manejo da doença fibrocística (Quadro 3). 0 SCS inclui 5 categorias: atividade geral, achados radiológicos, baqueteamento digital, crescimento e desenvolvimento, e complicações, graduadas com 0, 1 e 2 pontos, de acordo com o quadro clínico do paciente. 0 paciente gravemente acometido recebe 0 , enquanto que o individuo sem nenhum comprometimento pode receber até 2 pontos em cada categoria, somando um máximo de 10 pontos $^{(7)}$. A SCS foi aplicada em 45 pacientes, avaliados periodicamente durante um ano e meio, e os próprios autores reconheceram a limitada experiência na utilização desse escore.

Em 1973, Taussig criou o National Institute of Health Score ( $\mathrm{NIH})$, um escore clínico para avaliação de gravidade da FC, que se propõe objetivo e é definido pelo autor como simples, fácil e imediato. Para testar esse escore, Taussig avaliou 73 pacientes, entre cinco e 30 anos de idade, por um período de três a seis anos. Foram consideradas as complicações prevalentes, como pneumotórax, hemoptise e cirurgias, com significado prognóstico. 0 escore inclui avaliação pulmonar e geral, com subcategorias com pontuações específicas, somando o indivíduo gravemente acometido até 100 pontos. Dados detalhados da clínica, como gasometria, presença de comprometimento gastrointestinal e artropatia também são considerados na avaliação, mas não recebem pontuação (Quadro 4). A aderência do paciente ao tratamento também foi associada à identificação da gravidade da doença através desse escore, como forma de suprir o médico e a família de um preditor numérico de expectativa de vida, aumentando assim a sua participação nas terapêuticas multiprofissionais ${ }^{(8)}$.

Um ano depois, Chrispin \& Norman criaram o primeiro escore radiológico de avaliação de gravidade da FC, considerando o fato de que alterações no exame radiológico retratam a progressão da doença pulmonar e podem ser correlacionadas com a condição clínica do paciente (Quadro 5). Para a elaboração desse escore, de análise sistemática de imagens, os autores preocuparam-se em uniformizar os termos e descrever as cinco características radiológicas consideradas. A avaliação é baseada na divisão do radiograma pulmonar em quatro zonas e na classificação das imagens em três categorias, de acordo com a presença e gravidade das alterações ${ }^{(17)}$. Para a comprovação desse escore, dois examinadores avaliaram os radiogramas de 30

\begin{tabular}{|c|c|c|c|}
\hline CATEGORIAS & 2 & 1 & 0 \\
\hline atividades & $\begin{array}{c}\text { empenho atlético com } \\
\text { acompanhamento } \\
\text { normal }\end{array}$ & $\begin{array}{l}\text { freqüência escolar regular com } \\
\text { acompanhamento normal }\end{array}$ & \\
\hline & $\begin{array}{c}\text { atividade física } \\
\text { normal }\end{array}$ & máximo de 2 faltas no mês & \\
\hline Radiografia & normal & $\begin{array}{c}\text { marcas minimamente } \\
\text { aumentadas e enfisema }\end{array}$ & \\
\hline $\begin{array}{l}\text { baqueteamento } \\
\text { crescimento e }\end{array}$ & $\begin{array}{c}0 \text { a } 1+ \\
\text { peso e altura acima do }\end{array}$ & $\begin{array}{c}1 \text { a } 2+\text { sem cianose } \\
\text { peso e altura acima do }\end{array}$ & $\begin{array}{l}2+\text { e extensos } \\
\text { peso e altura } \\
\text { abaixo }\end{array}$ \\
\hline desenvolvimento & percentil 25 & percentil 3 & do percentl 3 \\
\hline Complicações & sem & transitórias & fixas \\
\hline
\end{tabular}

Quadro 3. Escore de Cooperman - SCS 


\begin{tabular}{|lc|}
\hline PULMÃo & PONTUAÇÃO \\
\hline raio-X & $01-17$ \\
função pulmonar & $01-17$ \\
exacerbação pulmonar & $03-05$ \\
Pneumotórax & $03-05$ \\
Hemoptise & $04-07$ \\
cirurgia pulmonar & $02-07$ \\
cor pulmonale & $03-05$ \\
ausculta pulmonar & $01-09$ \\
tosse e expectoração & $01-03$ \\
\hline TOTAL & 75 \\
\hline GERAL & PONTUAÇÃO \\
\hline Peso & $01-06$ \\
Atividade & $01-10$ \\
Atitude & $01-09$ \\
\hline TOTAL & 25 \\
\hline COMPLICAÇÕES & CARACTERiSTICAS \\
\hline gasometria & PO ${ }_{2}$ \\
& obstrução \\
gastrointestinais & má absorção \\
& anormalidades \\
& pólipos nasais \\
outras & sinusites \\
& infertilidade masculina \\
\hline Quadro elaborado a partir do artigo original de Taussig, 1973) \\
\hline
\end{tabular}

Quadro 4. Escore de Taussig - NIH

\begin{tabular}{|lccc|}
\hline Caracteristicas & Ausente & Leve Intensa \\
\hline tórax & 0 & 1 & 2 \\
abaulamento esternal & 0 & 1 & 2 \\
rebaixamento do diafragma & 0 & 1 & 2 \\
cifose espinhal & 0 & & \\
espessamento das paredes & & & \\
brônquicas & & & 2 \\
zona superior direita & 0 & 1 & 2 \\
zona superior esquerda & 0 & 1 & 2 \\
zona inferior direita & 0 & 1 & 2 \\
zona inferior esquerda & 0 & 1 & 2 \\
consolidações esparsas & & & \\
zona superior direita & 0 & 1 & 2 \\
zona superior esquerda & 0 & 1 & 2 \\
zona inferior direita & 0 & 1 & 2 \\
zona inferior esquerda & 0 & 1 & 2 \\
imagens anelares & & & \\
zona superior direita & 0 & 1 & 2 \\
zona superior esquerda & 0 & 1 & 2 \\
zona inferior direita & 0 & 1 & 2 \\
zona inferior esquerda & 0 & 1 & 2 \\
opacidades grandes & & & \\
zona superior direita & 0 & 1 & 2 \\
zona superior esquerda & 0 & 1 & 2 \\
zona inferior direita & 0 & 1 & 2 \\
zona inferior esquerda & 0 & 1 & 2 \\
\hline (Quadro elaborado a partir do artigo original de Chrispin \&t \\
\hline
\end{tabular}

Quadro 5. Escore de Chrispin \& Norman crianças com FC, e reavaliaram o mesmo exame 20 meses depois, para comparação dos resultados.

Mesmo com vários EGFC sendo aplicados, persistiam as discussões e questionamentos quanto à variabilidade na gravidade das manifestações clínicas, correlação das mudanças radiológicas com a doença pulmonar e eficácia e efetividade dos escores existentes. Esse fato incentivou Brasfield et. al. a elaborarem, em 1979, um novo escore radiológico, que foi comprovado através da avaliação de 643 imagens de 118 pacientes por um radiologista pediátrico e dois clínicos, separadamente ${ }^{(10)}$. Esse estudo teve também a preocupação de constatar a reprodutibilidade do sistema, e sua associação com parâmetros clínicos e com a função pulmonar. 0 escore apresenta cinco categorias que podem ser pontuadas de 0 a 5 , recebendo a pontuação máxima de 25 as alterações radiológicas mais graves (Quadro 6).
A criação de sete escores, radiológicos e clínicos, em um período de questionamento sobre o aumento da sobrevida e importância da precocidade do diagnóstico na FC, motivou Piepszi et. al. ${ }^{(18)}$ a discutirem a criação de um escore cintilográfico. Foram avaliadas 285 cintilografias, de 111 pacientes de dois meses de idade a 20 anos, por dois examinadores. A avaliação considerou cada pulmão dividido em três partes, com atribuição de 0 a 2 pontos, dependendo da gravidade da alteração. A obtenção de um total de 12 pontos retrata um acometimento grave na perfusão. Apesar desse estudo ressaltar a grande importância da avaliação cintilográfica, não descreveu detalhadamente um sistema de quantificação na FC.

No ano seguinte, tentando considerar as falhas dos EGFC anteriores ${ }^{(5,8)}$ e contribuir com o período de grande atenção dada à avaliação dos 


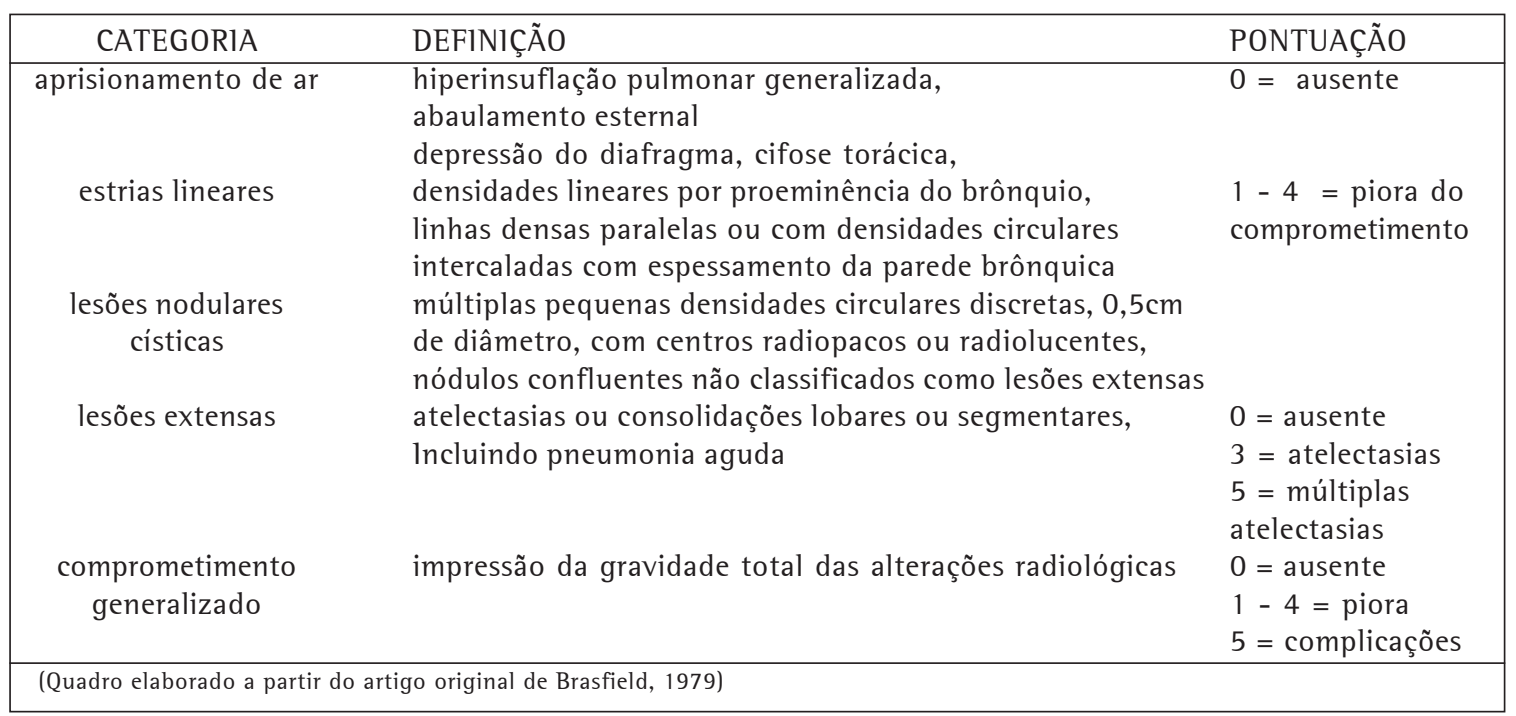

Quadro 6. Escore de Brasfield

parâmetros clínicos na FC, Huang et. al. ${ }^{(19)}$ publicaram o sistema de pontuação criado no serviço médico onde trabalhavam, utilizado para avaliar a resposta terapêutica aos diferentes regimes de antibióticos propostos na época ${ }^{(20,21)}$. Para validar o score de Huang, dois de seus quatro autores avaliaram 22 pacientes com FC através desse sistema, e comprovaram estatisticamente sua consistência e confiabilidade. Esse escore tem 20 itens, valendo cinco pontos cada: 10 elementos clínicos, 5 radiológicos e 5 de função pulmonar (Quadro 7). Quanto menor a pontuação, maior a gravidade. Também considera cinco complicações características da doença, pontuadas de acordo com a gravidade ( 5 pontos para o estado brando e 10 para a complicação grave). A peculiaridade desse escore é a sua aplicação antes e depois de uma conduta terapêutica, para identificar a piora ou melhora do quadro clínico diante da intervenção ${ }^{(19)}$.

Em 1982, uma modificação do escore de Chrispin \& Norman $^{(17)}$ foi proposta por Van der Put et. al. ${ }^{(22)}$, a qual incluiu 4 pontos a mais no escore original pertinente à imagem de alargamento hilar. Esse escore, chamado C-N score(22), foi elaborado para a investigação da idade do aparecimento dos sinais radiológicos típicos da FC, para o acompanhamento da doença, e para comparar o escore dos fibrocísticos com o escore de pacientes com alguma doença pulmonar obstrutiva e de crianças com provável cardiopatia. Essa comparação entre os escores não evidenciou as características de hiperinsuflação e imagens lineares como sendo específicas para a FC, ao contrário do que ocorreu com as imagens anelares e condensações $^{(22)}$.

Em 1987, uma nova adaptação de escores já existentes foi proposta por Lewiston ${ }^{(23)}$. Esse novo escore constituiu o escore radiológico de Brasfield $^{(10)}$ como substituto da categoria de avaliação do radiograma do escore clínico de Shwachman ${ }^{(5)}$, e foi chamado escore S-B. Foi elaborado em um período no qual instituições especializadas, pesquisas e triagens de efeitos terapêuticos passaram a usar os escores existentes e a questionar as variações de avaliação entre os examinadores. Esse novo EGFC foi criado para aplicação em estudos multicêntricos, e sua reprodução de critérios foi comprovada pela pequena variação no resultado da avaliação de 41 pacientes por cinco examinadores ${ }^{(23)}$.

Mesmo com o uso sistemático de sistemas de escores clínicos ${ }^{(5,6,8)}$, radiológicos ${ }^{(17,10)}$, e com provas de função pulmonar e culturas de escarro, foi desenvolvido o primeiro escore de avaliação tomográfica de alta-resolução, o chamado escore de Nathanson, para maior precisão na avaliação pulmonar e determinação da condição e do local 


\begin{tabular}{|c|c|c|c|c|c|c|c|c|c|c|c|}
\hline \multirow{2}{*}{$\begin{array}{l}\text { AVALIAÇÂA } \\
\text { CLÍNICO (50) }\end{array}$} & \multicolumn{10}{|c|}{ PONTUAÇÂO } & \multirow{2}{*}{$\begin{array}{c}\text { TOTAL } \\
\text { PERCENTUAL FINAL }\end{array}$} \\
\hline & \multicolumn{5}{|c|}{ PRÉ -TRATAMENTO } & \multicolumn{5}{|c|}{ PÓS -TRATAMENTO } & \\
\hline peso & 5 & 4 & 3 & 2 & 1 & 5 & 4 & 3 & 2 & 1 & \\
\hline atividades & 5 & 4 & 3 & 2 & 1 & 5 & 4 & 3 & 2 & 1 & SOMA DOS TOTAIS \\
\hline tosse & 5 & 4 & 3 & 2 & 1 & 5 & 4 & 3 & 2 & 1 & PARCIAIS \\
\hline apetite & 5 & 4 & 3 & 2 & 1 & 5 & 4 & 3 & 2 & 1 & \\
\hline ausculta pulmonar & 5 & 4 & 3 & 2 & 1 & 5 & 4 & 3 & 2 & 1 & DS - AS $\times 100$ \\
\hline freqüência respiratória & 5 & 4 & 3 & 2 & 1 & 5 & 4 & 3 & 2 & 1 & $\overline{A S}$ \\
\hline febre & 5 & 4 & 3 & 2 & 1 & 5 & 4 & 3 & 2 & 1 & - \\
\hline hemograma & 5 & 4 & 3 & 2 & 1 & 5 & 4 & 3 & 2 & 1 & $\mathrm{AS}=$ soma na alta \\
\hline culturas & 5 & 4 & 3 & 2 & 1 & 5 & 4 & 3 & 2 & 1 & $\mathrm{DS}=$ soma na admissão \\
\hline condição geral & 5 & 4 & 3 & 2 & 1 & 5 & 4 & 3 & 2 & 1 & \\
\hline RADIOLÓGICO (25) & & & & & & & & & & & COMPLICAÇÕES \\
\hline aprisionamento de ar & 5 & 4 & 3 & 2 & 1 & 5 & 4 & 3 & 2 & 1 & \\
\hline espessamento peribrônquico & 5 & 4 & 3 & 2 & 1 & 5 & 4 & 3 & 2 & 1 & pneumotórax \\
\hline nódulos/cistos & 5 & 4 & 3 & 2 & 1 & 5 & 4 & 3 & 2 & 1 & hemoptise \\
\hline segmentar/lobar atelectasias & 5 & 4 & 3 & 2 & 1 & 5 & 4 & 3 & 2 & 1 & $\begin{array}{l}\text { hematêmese falência } \\
\text { respiratória }\end{array}$ \\
\hline $\begin{array}{l}\text { impressão geral } \\
\text { TOTAL PARCIAL }\end{array}$ & 5 & 4 & 3 & 2 & 1 & 5 & 4 & 3 & 2 & 1 & $\begin{array}{c}\text { espessamento cardíaco/ } \\
\text { falência cardíaca congestiva }\end{array}$ \\
\hline \multicolumn{12}{|l|}{ FUNÇÃO PULMONAR (25) } \\
\hline capacidade vital & 5 & 4 & 3 & 2 & 1 & 5 & 4 & 3 & 2 & 1 & Pontuação de 5 - 10 \\
\hline FEV1/FVC & 5 & 4 & 3 & 2 & 1 & 5 & 4 & 3 & 2 & 1 & pré e pós tratamento \\
\hline MMEFR & 5 & 4 & 3 & 2 & 1 & 5 & 4 & 3 & 2 & 1 & \\
\hline RV/TLC & 5 & 4 & 3 & 2 & 1 & 5 & 4 & 3 & 2 & 1 & 5 = leve \\
\hline Vmáx 25\% VC & 5 & 4 & 3 & 2 & 1 & 5 & 4 & 3 & 2 & 1 & $10=$ grave \\
\hline \multicolumn{12}{|l|}{ TOTAL PARCIAL } \\
\hline
\end{tabular}

Quadro 7. Escore de Huang

de bronquiectasias e impactações mucóides na via aérea de pacientes com $\mathrm{FC}^{(24)}$. Para desenvolver esse escore, os exames de 28 pacientes com FC foram avaliados cegamente por dois radiologistas e um pneumologista pediátrico, que aplicaram também o escore de Shwachman ${ }^{(5)}$, o escore de Brasfield $^{(10)}$, e a prova de função pulmonar em 19 pacientes. Nesse escore, a tomografia de tórax é pontuada através da divisão do pulmão em doze zonas, em que cada uma recebe uma pontuação separadamente. A bronquiectasia é classificada de 0 a 5 pontos, de acordo com sua gravidade, e a presença de muco impactado determina 1 ponto. Uma maior pontuação corresponde a uma maior gravidade.

A avaliação tomográfica no acompanhamento da doença pulmonar foi ainda mais discutida pelo estudo de Bhalla et. al. ${ }^{(25)}$. Considerando os escores radiológicos como imprecisos e subjetivos, criaram também um escore tomográfico para assessorar a avaliação do comprometimento pulmonar, os efeitos terapêuticos e a seleção de pacientes para transplante. As tomografias de quatorze pacientes, com idades entre 5 e 42 anos, foram analisadas retrospectivamente, por três radiologistas, através da técnica de quantificação morfológica proposta pelos autores, o chamado escore de Bhalla ${ }^{(25)}$. Esse escore apresentou resultados positivos e significativos pela sua pequena variação entre os examinadores, boa reprodutibilidade e alta correlação com a prova de função pulmonar. Sua pontuação é feita através de 9 categorias, com valor de 3 pontos cada, e a pontuação máxima é sinônimo de gravidade. 0 resultado final do escore deve ser subtraído de 25, e quanto menor o resultado, mais grave o paciente (Quadro 8).

Os avanços no diagnóstico e tratamento da FC e a falta de um sistema de avaliação radiológica realmente sensivel e reprodutivel para a quantificação da doença pulmonar direcionaram a criação de outro escore, o escore de Wisconsin ${ }^{(26)}$. Esse escore radiológico foi estruturado em três 
fases: elaboração de seus atributos e pontuação, comparação com o escore de Brasfield(10), e validação. Ele é formado por 6 atributos principais e um total de 24 componentes individuais com pontuação específica, com variação de 0 a 100 pontos no total (Quadro 9). Recebe uma maior pontuação o paciente com doença pulmonar mais grave $^{(26)}$.

Como os escores radiológicos anteriores exigiam a avaliação de uma junta médica para a graduação do envolvimento pulmonar do paciente, em 1994 Conway propôs o escore de Northern ${ }^{(27)}$, que permite que o procedimento seja feito por um único examinador. Em seu estudo, 45 exames radiológicos foram avaliados por dez clínicos, que compararam o novo escore com outros escores radiológicos ${ }^{(10,17)}$. 0 escore de Northern ${ }^{(27)}$ apresentou uma melhor concordância entre os examinadores, independentemente da avaliação da imagem radiológica de perfil (Quadro 10).

Pela necessidade de avaliação de adultos e maior clareza nos critérios de pontuação, Matouk et. al. ${ }^{(29)}$ modificaram o escore de Huang ${ }^{(19)}$, detalhando melhor alguns de seus itens, mas respeitando a estrutura original. Alta consistência e correlação com o volume expiratório forçado no primeiro segundo $\left(\mathrm{FEV}_{1}\right)$ foram resultados estatisticamente significativos na aplicação desse escore em estudo com 109 adultos com $\mathrm{FC}^{(28)}$, e sua utilização na avaliação do efeito de intervenções terapêuticas ficou demonstrada dois anos depois ${ }^{(29)}$.

\begin{tabular}{|c|c|c|c|c|}
\hline CATEGORIA & 0 & 1 & 2 & 3 \\
\hline $\begin{array}{c}\text { GRAVIDADE } \\
\text { DA BRONQUIECTASIA }\end{array}$ & ausente & $\begin{array}{c}\text { Leve } \\
\text { (luz sutilmente } \\
\text { maior que vaso } \\
\text { adjacente) }\end{array}$ & $\begin{array}{c}\text { Moderada } \\
\text { (luz } 2 / 3 \times \\
\text { maior que o vaso } \\
\text { adjacente) }\end{array}$ & $\begin{array}{c}\text { Grave } \\
\text { (luz 3x } \\
\text { maior que o } \\
\text { vaso adjacente) }\end{array}$ \\
\hline $\begin{array}{l}\text { ESPESSAMENTO } \\
\text { PERIBRÔNQUICO }\end{array}$ & ausente & $\begin{array}{l}\text { Leve } \\
\text { (espessamento da } \\
\text { parede igual vaso) }\end{array}$ & $\begin{array}{l}\text { moderado } \\
\text { (espessamento } \\
\text { maior/dobro } \\
\text { do vaso) }\end{array}$ & $\begin{array}{l}\text { Grave (espessamento } 2 x \\
\text { maior que o vaso) }\end{array}$ \\
\hline $\begin{array}{l}\text { EXTENSÃO DA } \\
\text { BRONQUIECTASIA (n }{ }^{\circ} \\
\text { segmentos broncopulmonar) }\end{array}$ & ausente & $1-5$ & $6-9$ & $>9$ \\
\hline $\begin{array}{l}\text { EXTENSÃO DE ROLHAS DE } \\
\text { MUCO (nº segmentos } \\
\text { broncopulmonar) }\end{array}$ & ausente & $1-5$ & $6-9$ & $>9$ \\
\hline $\begin{array}{c}\text { ABCESSOS OU SACULAÇÕES } \\
\left(n^{\circ} \text { segmentos }\right. \\
\text { broncopulmonar })\end{array}$ & ausente & $1-5$ & $6-9$ & $>9$ \\
\hline $\begin{array}{c}\text { GENERALIDADES DA } \\
\text { DIVISÃO BRONQUIAL } \\
\text { ENVOLVIDA } \\
\text { (bronquiectasia/rolha) }\end{array}$ & ausente & $\begin{array}{l}\text { acima da } \\
4^{\mathrm{a}} \text { geração }\end{array}$ & $\begin{array}{l}\text { acima da } \\
5^{\text {a }} \text { geração }\end{array}$ & $\begin{array}{c}\text { acima da } 6^{\mathrm{a}} \\
\text { geração e distal }\end{array}$ \\
\hline NÚMERO DE BOLHAS & ausente & $\begin{array}{l}\text { unilateral } \\
\text { (não > 4) }\end{array}$ & $\begin{array}{l}\text { bilateral } \\
\text { (não > } 4 \text { ) }\end{array}$ & $>4$ \\
\hline ENFISEMA( $n^{\circ}$ segmentos BP) & ausente & $1-5$ & $>5$ & \\
\hline COLAPSO / CONSOLIDAÇÃO & ausente & subsegmental & segmental lobar & \\
\hline
\end{tabular}

Quadro 8. Escore de Bhalla 
0 escore clínico mais recente é o Cystic Fibrosis clinical score, criado por Kanga et. al. ${ }^{(12)}$, em um estudo que correlacionou sinais e sintomas com deterioração da função pulmonar em fibrocísticos ${ }^{(30)}$. Segundo os autores, é um escore de avaliação da exacerbação pulmonar aguda, que pretende identificar a agudização da doença, predizer a melhora ou piora da função respiratória e avaliar os efeitos terapêuticos. Nesse estudo, ele é definido como um escore simples, de fácil aplicação e baixo custo, que incorpora dados da avaliação de rotina do paciente, com variáveis clínicas comuns, pequena variabilidade entre examinadores, alta correlação com a prova de função pulmonar (VEF ${ }_{1}$ e capacidade vital forçada - (VF) e que detecta mudanças clínicas diárias. Foi comprovado pela avaliação de 130 pacientes de cinco a dezessete anos em exacerbação pulmonar aguda em estudo multicêntrico prospectivo. Inclui 5 sintomas comuns: tosse, produção de secreção, perda de apetite, dispnéia e perda de energia, e 5 sinais físicos: temperatura, peso, freqüência respiratória, chiado e estertoração. 0 paciente pode receber de 1 a 5 pontos em cada critério e a maior pontuação ocorre no indivíduo mais acometido. Uma queda de 15 pontos no escore sugere melhora clínica e o aumento de 10 a 15 pontos indica necessidade de hospitalização, segundo os autores.

\section{ANÁLISE CRÍTICA}

Os objetivos, aplicações e propostas dos EGFC merecem atenção na comunidade científica, visto que na FC há grande dificuldade na apreciação de

\begin{tabular}{|c|c|c|c|c|}
\hline HIPERINSUFLAÇÃO & NENHUM & NORMAL / PRESENTE & MÉDIO & GRAVE \\
\hline contorno diafragmático & - & 0 & 0.3 & 1.0 \\
\hline ar retroesternal & - & 0 & 0.3 & 1.0 \\
\hline posição cardíaca & 0 & 1.0 & - & - \\
\hline abaulamento esternal & 0 & 1.0 & - & - \\
\hline cifose & 0 & 1.0 & - & - \\
\hline ESPESSAMENTO & \multicolumn{3}{|c|}{ CENTRAL } & \\
\hline PERIBRÔNQUICO & MÉDIO & MODERAD0 & GRAVE & NENHUM \\
\hline \multicolumn{5}{|l|}{ PERIFERIA } \\
\hline MÉD10 & 0.40 & 0.50 & 0.65 & - \\
\hline MODERADO & - & 0.75 & 0.85 & - \\
\hline GRAVE & - & - & 1.00 & - \\
\hline NENHUM & 0.10 & 0.20 & 0.30 & 0 \\
\hline BRONQUIECTASIAS & NENHUMA & MÉDIA & GRAVE & \\
\hline pontuar cada quadrante & 0 & 0.50 & 1.0 & \\
\hline $\begin{array}{c}\text { OPACIDADES NODULARES } \\
\text { OU RAMIFICADAS }\end{array}$ & NENHUMA & MÉDIA & GRAVE & \\
\hline pontuar cada quadrante & 0 & 0.50 & 1.0 & \\
\hline OPACIDADES DEFINIDAS & AUSENTE & PRESENTE & & \\
\hline 1 lobo & - & 0.20 & & \\
\hline 2 lobos & - & 0.40 & & \\
\hline 3 lobos & - & 0.60 & & \\
\hline 4 lobos & - & 0.80 & & \\
\hline 5 lobos & - & 1.0 & & \\
\hline ATELECTASIAS & PARCIAL & COMPLETA & & \\
\hline nenhum lobo afetado & 0 & 0 & & \\
\hline 1 lobo & 0.0825 & 0.333 & & \\
\hline 2 lobos & 0.165 & 0.667 & & $\#=100$ pontos \\
\hline 3 lobos & 0.2475 & 1.00 & & \\
\hline 4 lobos & 0.333 & $\#$ & & \\
\hline 5 lobos & 0.415 & $\underline{\#}$ & & \\
\hline
\end{tabular}

Quadro 9. Escore de Wisconsin 
seus estágios de gravidade e os escores constituem um instrumento importante para a avaliação e controle do cuidado ao paciente fibrocístico.

Cada EGFC surgiu em um contexto histórico específico da doença e, com isso, tentou suprir as necessidades da época.

Na década de 1950, período de discussões sobre a necessidade de um instrumento de avaliação clínica e prognóstica que permitisse a comparação entre os doentes, definisse a gravidade da doença e assessorasse a terapêutica, Mclntosh propôs um sistema simples de avaliação clínica ${ }^{(15)}$. Mas foi o escore de Shwachman ${ }^{(5)}$ o primeiro escore clínico realmente elaborado. Apesar de ser o mais utilizado na comunidade médica, recebe muitas críticas, entre elas a subjetividade e globalidade de seus critérios, a falta de sistematização para sua aplicação, o fato de que não ressalta a avaliação do sistema respiratório, por desconsiderar o teste de função pulmonar e as complicações decorrentes da progressão da doença, e por ter sido estruturado a partir de evidências clínicas em crianças $^{(6-8,11,12)}$. Esse escore é até hoje reconhecido por sua importância no entendimento da doença ${ }^{(15,5)}$ e foi adaptado por Doershuk et. al. visando uma maior objetividade e a inclusão de adolescentes e adultos na avaliaçã $0^{(6)}$. Modificado, é ainda considerado muito subjetivo ${ }^{(7,8)}$, mas mesmo assim tem muitos adeptos. Já o escore de Cooperman, uma simplificação dos escores existentes na época ${ }^{(7)}$, raramente é utilizado.

Ao mesmo tempo em que a subjetividade era exaustivamente questionada e ressaltava-se a necessidade de um escore clínico compreensivo e objetivo que contribuísse com o conhecimento da evolução e tratamento precoce da doença ${ }^{(7)}$, houve o aumento na sobrevida dos pacientes e o surgimento das complicações decorrentes disso. Único fundamentado nessas complicações clínicas, o escore de Taussig, o NIH score ${ }^{(8)}$, é reconhecido por assessorar no prognóstico, acompanhar a evolução da doença, ${ }^{(32)}$ e por tentar solucionar os problemas apontados no escore de Shwachman. Mas alguns autores criticam a sua complexidade ${ }^{(33)}$, a dependência de trabalho em conjunto com a família, o fato de superestimar alguns elementos clínicos raros e menosprezar outros ${ }^{(31)}$, a exclusão de menores de cinco anos e a não avaliação das mudanças diárias do quadro clínico(12). Sua proposta de informar o paciente quanto ao prognóstico, a falta de especificação na graduação de suas variáveis ${ }^{(33,31)}$ e a grande variabilidade da avaliação pulmonar ${ }^{(34)}$ também são focos de discussão. Mesmo assim, Huang ${ }^{(19)}$ considerou o $\mathrm{NIH}$ score mais compreensível que o escore de Shwachman.

Huang divulgou o escore utilizado em seu serviço $^{(19,20,21)}$, o qual além de ser considerado como bastante completo, aborda uma preocupação médica constante: a de quantificar as respostas aos diferentes tratamentos ${ }^{(19,20,21)}$. Esse escore avalia, adicionalmente, a prova de função pulmonar, a falência respiratória hipercápnica e hipoxêmica e outras complicações pulmonares não pontuadas em outros escores, e tem sido utilizado em alguns estudos ${ }^{(28,29)}$. Nessa mesma linha, Kanga et. al. ${ }^{(12)}$ criaram um escore com enfoque preventivo, baseado na avaliação

\begin{tabular}{|c|c|c|}
\hline CLASSIFICAÇÃO & ALTERAÇÕES RADIOLÓGICAS & PONTUAÇÃO \\
\hline normal & sem comprometimento pulmonar evidente & 0 \\
\hline leve & $\begin{array}{l}\text { mínimo aumento de sinais lineares ou lesões nodulares } \\
\text { císticas acima de } 0,5 \mathrm{~cm} \text { de diâmetro }\end{array}$ & 1 \\
\hline moderada & $\begin{array}{l}\text { sinais lineares mais pronunciados } \\
\text { e lesões nodulares císticas mais difusas } \\
\text { proeminente aumento dos sinais lineares, }\end{array}$ & 2 \\
\hline grave & $\begin{array}{l}\text { lesões nodulares císticas profusas } \\
\text { extensas áreas de colapso/consolidação }\end{array}$ & 3 \\
\hline muito grave & $\begin{array}{l}\text { pequena ou nenhuma área de pulmão normal visível, } \\
\text { infiltração densa }\end{array}$ & 4 \\
\hline
\end{tabular}

Quadro 10. Escore de Northern 
diária de sinais e sintomas apresentados pelos pacientes, para detectar a exacerbação pulmonar aguda, comparar a resposta terapêutica, diferenciar a intensidade da doença e controlar a função respiratória. Embora ainda não tenham sido publicados estudos utilizando esse escore, ele já pode ser considerado de grande importância por retratar a situação atual da FC, na qual os escores existentes estão sendo questionados ${ }^{(11)}$, importantes descobertas terapêuticas estão sendo feitas, e em que há uma preocupação universal em relação à aderência do paciente ao tratamento e à precocidade do diagnóstico e da intervenção.

Alguns estudos mostraram alta correlação do exame radiológico com a clínica e a prova de função pulmonar de pacientes com $\mathrm{FC}$ (36,37,38,39). Assim, os escores radiológicos também são reconhecidamente importantes para avaliação desses pacientes ${ }^{(35)}$.

0 primeiro escore radiológico específico foi o de Chrispin \&t Norman ${ }^{(17)}$, decorrente de estudos que já identificavam a variedade no comprometimento pulmonar dos pacientes e indicavam o exame radiológico como importante indicador da evolução da doença pulmonar ${ }^{(40,41)}$. Seus autores procuraram protocolar de forma simples alterações específicas definidas previamente, que fossem compatíveis com a gravidade e correlacionadas com a clínica. No entanto, o escore foi questionado por não considerar a sua reprodutibilidade ${ }^{(10,26)}$. Já o escore radiológico de Brasfield ${ }^{(10)}$, de reprodutibilidade comprovada, e de alta correlação com a prova de função pulmonar e com o prognóstico, foi considerado pelos médicos como um bom recurso no manejo da $\mathrm{FC}^{(38)}$. Ambos são utilizados nos grandes centros de FC e nas pesquisas, apesar da existência de críticas subseqüentes, como a falta de flexibilidade no escore de Brasfield ${ }^{(10)}$ e de discriminação da gravidade no de Chrispin \& Norman ${ }^{(27)}$. Para Weatherly et. al. ${ }^{(26)}$ o escore de Wisconsin reflete melhor a doença pulmonar leve quando comparado ao escore de Brasfield. Já o escore radiológico de Northern é considerado simples e prático ${ }^{(35)}$, e permite que uma única pessoa avalie o radiograma ${ }^{(27)}$.

Assim como Doershuk ${ }^{(6)}$ modificou o escore clínico de Shwachman ${ }^{(5)}$, também foram feitas algumas adaptações nesses escores radiológicos.
Van der Put ${ }^{(22)}$ alterou o escore de Chrispin $\&$ Norman ${ }^{(17)}$ para aplicação em seu estudo e o estudo de Lewiston ${ }^{(23)}$ substituiu a avaliação radiológica do escore de Shwachman pela proposta de Brasfield ${ }^{(10)}$.

Apesar de o exame radiológico ser barato, de fácil realização e da pouca exposição do paciente à radiação, o advento da tomografia computadorizada permitiu uma melhor visualização das estruturas pulmonares e conseqüente precocidade na detecção de alterações das vias aéreas $^{(24)}$, além de envolver termos menos subjetivos e imprecisos ${ }^{(25)}$. Para uma análise minuciosa de entidades patologicamente distintas e com importante valor prognóstico, dois escores tomográficos foram criados: o Bhalla score ${ }^{(25)}$ e o Nathanson score ${ }^{(24)}$ (alta-resolução), ambos direcionados à condução de regimes terapêuticos, indicação de intervenção cirúrgica e seleção de pacientes para transplante.

Em meio a escores clínicos, radiológicos e tomográficos, uma proposta de avaliação cintilográfica também foi sugerida por Piepsz et. al. ${ }^{(18)}$, porém sem definição clara dos critérios cintilográficos e sem apresentar um escore propriamente dito. Mas assim como outros, o estudo ressalta a necessidade da análise do conjunto de exames e dos recursos tecnológicos para uma boa caracterização do comprometimento respiratório ${ }^{(35,39)}$, o que permite uma compreensão mais ampla da doença, de sua evolução, controle, terapêutica e prognóstico, elementos primordiais para qualquer centro de atendimento da FC.

A falta de um manual de orientação dos escores, com a grade de pontuação e forma de aplicação como apresentam Huang ${ }^{(19)}$ e Wisconsin (26) é outra consideração importante e que vai ao encontro de questões como a variabilidade e a reprodutibilidade dos escores, pois mesmo que alguns pareçam ser "auto-explicativos", a execução de forma correta parece ficar restrita ao serviço onde o EGFC foi criado e principalmente aos seus autores. A preocupação com a padronização e validação dos escores existentes é atual e vem sendo discutida, estudada e comprovada em trabalhos recentes ${ }^{(28,29,42)}$.

Apesar de todas as críticas, preocupações e controvérsias, os EGFC são considerados instrumentos acessiveis, de aplicação relativamente simples, e importantes no entendimento da doença 
e de sua evolução. Foram elaborados para ser preditores da evolução da doença, estabelecer a velocidade de sua progressão, identificar o quadro clínico, estimar a necessidade de intervenção, detectar respostas terapêuticas, e selecionar pacientes para cuidados especiais e imediatos. No entanto, ainda necessitam de definições mais precisas e medidas mais objetivas, principalmente no que se refere ao acompanhamento da função pulmonar, cuja deterioração é responsável pela maioria dos óbitos na FC.

\section{CONCLUSÃO}

Embora vários trabalhos nacionais $\mathrm{e}$ internacionais tenham mostrado que não existe associação entre as características genotípicas e fenotípicas na FC, as características clínicas e as mutações têm sido correlacionadas com a gravidade. A procura de marcadores de gravidade, clínicos e laboratoriais, tem sido uma preocupação constante na literatura médica no último século, como descrito no decorrer desse estudo, conforme os avanços no estudo da fisiopatogenia da FC. Isso impulsionou a criação de métodos de graduação do acometimento multissistêmico desses pacientes: os escores de avaliação da gravidade. Uma melhor compreensão da doença é hoje um fato, decorrente de meio século de estudos e pesquisas, e os EGFC participaram desse processo, caracterizando a evolução da doença, indicando a gravidade, direcionando o prognóstico, realizando a mensuração da eficácia e efetividade das intervenções. Com isso, são instrumentos de qualificação e quantificação das diferentes terapêuticas e mais um recurso disponível para a equipe multiprofissional dos centros de tratamento da FC, que deve selecionar o mais adequado e usá-lo na rotina de atendimento. Além disso, a progressão no conhecimento das características genotípicas e fenotípicas das cerca de mil mutações da FC, somada aos avanços dos recursos dos exames complementares (tomografia, cintilografia, ressonância magnética, etc), deverá propiciar, em futuro próximo, a modificação dos escores existentes e a criação de novos EGFC.

\section{REFERÊNCIAS}

1. Robinson P. Cystic fibrosis. Thorax. 2001;56:237-41.

2. Mitchell 1, Nakielna E, Tullis E, Adair C. Cystic fibrosis. Chest. 2000;118:80-4.
3. Zach MS, Oberwaldner B. Chest physiotherapy - the mechanical approach to antiinfective therapy in cystic fibrosis. Infection. 1987;15:381-4.

4. Döring G, Cönway SP, Heijerman HGM, Hodson ME, Hoiby N, Smyth A, et al. Antibiotic therapy against Pseudomonas aeruginosa in cystic fibrosis: a European consensus. Eur Respir J. 2000;16:749-67.

5. Shwachman H, Kulczycki LL. Long term study of one hundred five patients with cystic fibrosis. Am J Dis Child. 1958;96:6-15.

6. Doeurshuk CF, Matthews LW, Tucker AS, Nudelman H, Eddy G, Wise M, et al. A 5 year clinical evaluation of a therapeutic program for patients with cystic fibrosis. J Pediatr. 1964;65:677-93.

7. Cooperman EM, Park M, McKee J, Assad JP. A simplified cystic fibrosis scoring system (a preliminary report). CMAJ. 1971;105:580-1.

8. Taussig LM, Kattwinkel J, Friedewald WT, di Sant'Agnese PA. A new prognostic score and clinical evaluation system for cystic fibrosis. J Pediatr. 1973;82:380-90.

9. Wood RE. Cystic fibrosis: diagnosis, treatment and prognosis. South Med J. 1976;72:189-202.

10. Brasfield D, Hicks G, Soong S, Tiller RE. The chest roentgenogram in cystic fibrosis: a new scoring system. Pediatrics. 1979;63:24-9.

11. Taussig LM. The score is.... [editorial]. Pediatr Pulmonol. 1994;17:279-80.

12. Kanga J, Kuhn R, Craigmyle L, Haverstock D, Church D. Cystic fibrosis clinical score: a new scoring system to evaluate acute pulmonary exacerbation. Clin Ther. 1999;21:1343-56.

13. Huang NN, Macri CN, Girone J, Sproul A. Survival of patients with cistic fibrosis. Am J Dis Child. 1970, 120:289-95.

14. Jaffé A, Bush A. Cystic fibrosis: a review of the decade. Monaldi Arch Chest Dis. 2001;56:240-7.

15. Mclntosh R. Cystic fibrosis of the pancreas in patientes over 10 years of age. Acta Paediatr. 1954;43(Suppl 100):467.

16. Royce SW. Symposium on fibrocystic disease of the pancreas: report of the 18th Ross pediatric research conference held in lwoa city, Sept. 30-0ct. 1, 1955, Columbus, Ohio. Columbus, Ohio: Ross Laboratories; 1955.

17. Chrispin AR, Norman AP. The systematic evaluation of the chest radiograph in cystic fibrosis. Pediatr Radiol. 1974;2:101-6.

18. Piepsz A, Wetzburger C, Spehi M, Machin D, Dab 1, Ham $H R$, et al. Critical evaluation of lung scintigraphy in cystic fibrosis: study of 113 patients. Clin Sci. 1980;134:1195-8.

19. Huang NN, Keith HH, Palmer J, Hsuan F. A scoring system for short-term evaluation of patients with cystic fibrosis: a possible means for assessment of antibiotic efficacy. In: Warwick WJ, editor. 1,000 years of cystic fibrosis collected papers. Minessota: University Dept of Pediatrics Medical School in cooperation with International Cystic Fibrosis Association, National Heart, Lung and Blood Institute and Fogarty International Center; 1981. p.207-16.

20. Huang NN, Laraya-Cuasay LR, Yasmin N, Keith HH. Efficacy of sisomicin in patientes with cystic fibrosis. Infection. 1976;(Suppl 4):465. 
21. Huang NN, Laraya-Cuasay LR, Yasmin N. Cinical experience with amikacin in patients with cystic fibrosis. Am J Med. 1976 June;(Suppl):186.

22. van der Put JM, Meradji M, Danoesastro D, Kerrebijn KF. Chest radiographs in cystic fibrosis. A follow-up study with application of a quantitative system.Pediatr Radiol. 1982;12:57-61.

23. Lewiston N, Moss R, Hindi R, Rubinstein S, Sullivan M. Interobserver variance in clinical scoring for cystic fibrosis. Chest. 1987;91:878-82.

24. Nathanson 1, Conboy K, Murphy S, Afshani E, Kuhn JP. Ultrafast computerized tomography of the chest in cystic fibrosis: a new scoring system.Pediatr Pulmonol. 1991;11:81-6.

25. Bhalla M, Turcios N, Aponte V, Jenkins M, Leitman BS, McCauley Dl, et al. Cystic fibrosis: scoring system with thin-section CT. Radiology. 1991;179:783-8.

26. Weatherly MR, Palmer CG, Peters ME, Green CG, Fryback $\mathrm{D}$, Langhough $\mathrm{R}$, et al. Wisconsin cystic fibrosis chest radiograph scoring system. Pediatrics. 1993;91:48895.

27. Conway SP, Pond MN, Bowler 1, Smith DL, Simmonds EJ, Joanes DN, et al. The chest radiograph in cystic fibrosis: a new score system compared with the Chrispin-Norman and Brasfield scores. Thorax. 1994;49:860-2.

28. Matouk E, Ghezzo RH, Gruber J, Hidvegi R, Gray-Donald $\mathrm{K}$. Internal consistency reliability and predictive validity of a modified N. Huang clinical scoring system in adult cystic fibrosis patients. Eur Respir J. 1997;10:200413.

29. Matouk E, Ghezzo RH, Gruber J, Hidvegi R, Gray-Donald K. Construct and longitudinal validity of a modified N. Huang clinical scoring system in adult cystic fibrosis patients. Eur Respir J. 1999;13:552-9.

30. Kanga J, Wilson J, Posson A. The efficacy of reported symptoms and physical findings in indentifying children with clinically significant deterioration of pulmonary function: a longitudinal study. Pediatr Pulmonol. 1990;(Suppl 5):258.
31. Huang NN. A new prognostic score and clinical evaluation system for cystic fibrosis. J Pediatr. 1973;82:389-90.

32. Willinski CL, Warwick WJ, Budd JR. Mortality and progression of the $\mathrm{NIH}$ clinical and prognostic score. Pediatr Pulmonol. 1990;5:259-60.

33. Matthew LW. A new prognostic score and clinical evaluation system for cystic fibrosis. J Pediatr. 1973;82:389.

34. Sockrider MM, Swank PR, Seilheimer DK, Schidlow DV. Measuring clinical status in cystic fibrosis: internal validity and variability of a modified $\mathrm{NIH}$ score. Pediatr Pulmonol. 1994;17:86-9.

35. Shale DJ. Chest radiology in cystic fibrosis: is scoring useful? Thorax. 1994;49:847.

36. Kraemer R, Rüdeberg A, Kläy M, Rossi E. Relationship between clinical conditions, radiographic findings and pulmonary functions in patients with cystic fibrosis. Helv Paediatr Acta. 1979;34:417-28.

37. Coates AL, Boyce P, Shaw DG, Godfrey S, Mearns M. Relationship between the chest radiograph, regional lung function studies, exercise tolerance, and clinical condition in cystic fibrosis. Arch Dis Child. 1981;56:106-11.

38. Wong EBK, Regnis J, Shnier RC, Bye PTP, Stewart MEB. The relationship between tests of lung function and three chest radiological scoring systems in patients with cystic fibrosis. Australas Radiol. 1993;37:265-9.

39. Sawyer SM, Carlin JB, DeCampo M, G Bowes. Critical evaluation of three chest radiograph scores in cystic fibrosis. Thorax. 1994;49:863-6.

40. Bodian M. Fibrocystic disease of the pancreas. London: Heinemann; 1952. p.135.

41. White H. Fibrocystic disease of the pancreas roentgen manifestations. Radiology. 1958;71:86.

42. Koscik RE, Kosorok MR, Farrell PM, Collins J, Peters ME, Laxova A, et al. Wisconsin cystic fibrosis chest radiograph scoring system: validation and standardization for application to longitudinal studies. Pediatr Pulmonol. 2000;29:457-67. 